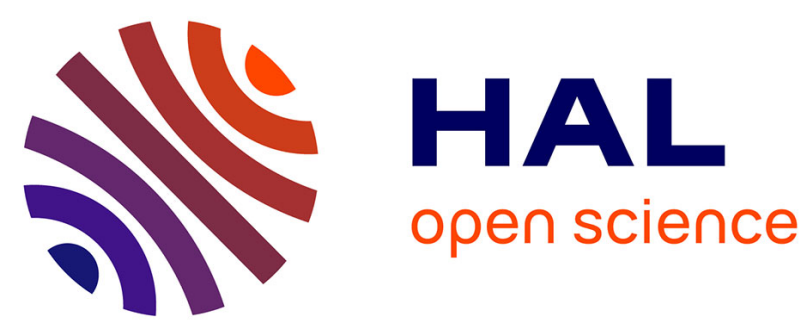

\title{
Elastic strains at interfaces in InAs/AlSb multilayer structures for quantum cascade lasers
}

Julien Nicolaï, Christophe Gatel, Bénédicte Warot-Fonrose, R. Teissier, A. N. Baranov, Cesar Magen, Anne Ponchet

\section{- To cite this version:}

Julien Nicolaï, Christophe Gatel, Bénédicte Warot-Fonrose, R. Teissier, A. N. Baranov, et al.. Elastic strains at interfaces in InAs/AlSb multilayer structures for quantum cascade lasers. Applied Physics Letters, 2014, 104 (3), pp.031907. 10.1063/1.4863035 . hal-01623721

\section{HAL Id: hal-01623721 \\ https://hal.science/hal-01623721}

Submitted on 18 Jun 2019

HAL is a multi-disciplinary open access archive for the deposit and dissemination of scientific research documents, whether they are published or not. The documents may come from teaching and research institutions in France or abroad, or from public or private research centers.
L'archive ouverte pluridisciplinaire HAL, est destinée au dépôt et à la diffusion de documents scientifiques de niveau recherche, publiés ou non, émanant des établissements d'enseignement et de recherche français ou étrangers, des laboratoires publics ou privés. 


\section{AIP Applied Pyysics Letters}

\section{Elastic strains at interfaces in InAs/AISb multilayer structures for quantum cascade}

lasers

J. Nicolai, Ch. Gatel, B. Warot-Fonrose, R. Teissier, A. N. Baranov, C. Magen, and A. Ponchet

Citation: Applied Physics Letters 104, 031907 (2014); doi: 10.1063/1.4863035

View online: http://dx.doi.org/10.1063/1.4863035

View Table of Contents: http://scitation.aip.org/content/aip/journal/apl/104/3?ver=pdfcov

Published by the AIP Publishing

\section{Articles you may be interested in}

High-resolution X-ray diffraction analysis of AlxGa1-xN/InxGa1-xN/GaN on sapphire multilayer structures:

Theoretical, simulations, and experimental observations

J. Appl. Phys. 115, 174507 (2014); 10.1063/1.4875382

Molecular beam epitaxy growth of high electron mobility InAs/AISb deep quantum well structure J. Appl. Phys. 114, 013704 (2013); 10.1063/1.4811443

Structural and transport characterization of AISb/InAs quantum-well structures grown by molecular-beam epitaxy with two growth interruptions

J. Vac. Sci. Technol. B 20, 1174 (2002); 10.1116/1.1468658

Molecular beam epitaxial growth of $\operatorname{lnAs} /(\mathrm{Al}, \mathrm{Ga}) \mathrm{Sb}$ quantum-well structures on germanium substrates Appl. Phys. Lett. 74, 3371 (1999); 10.1063/1.123348

Molecular beam epitaxial growth of InAs/AIGaAsSb deep quantum well structures on GaAs substrates J. Vac. Sci. Technol. B 16, 2644 (1998); 10.1116/1.590249

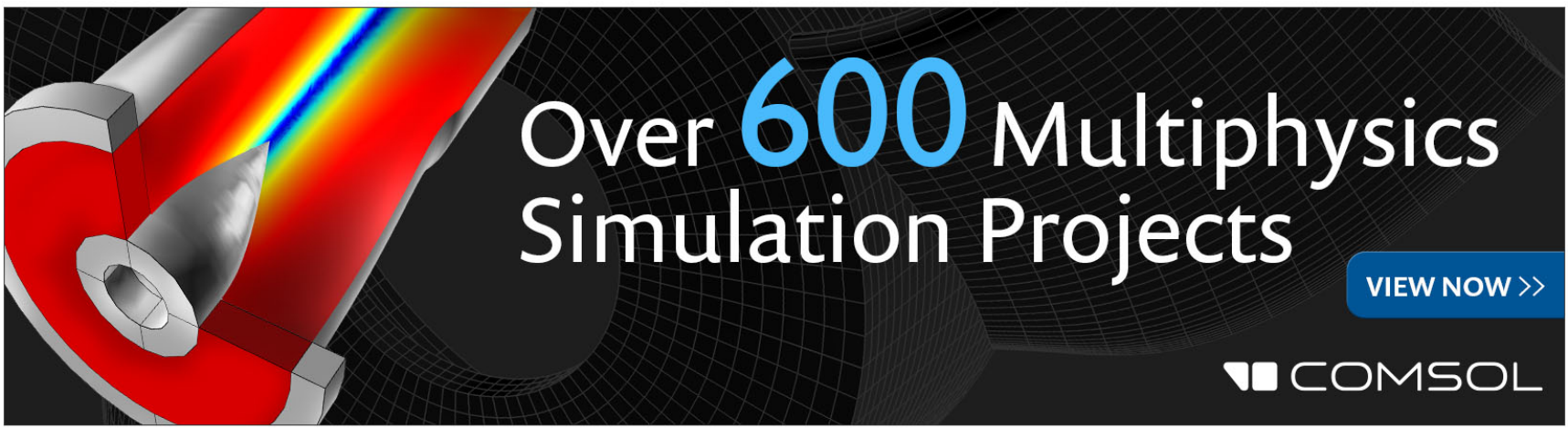




\title{
Elastic strains at interfaces in InAs/AISb multilayer structures for quantum cascade lasers
}

\author{
J. Nicolai, ${ }^{1}$ Ch. Gatel, ${ }^{1}$ B. Warot-Fonrose, ${ }^{1}$ R. Teissier, ${ }^{2}$ A. N. Baranov, ${ }^{2}$ C. Magen, ${ }^{3}$ \\ and A. Ponchet ${ }^{1}$ \\ ${ }^{1}$ CEMES CNRS-UPR 8011, Université de Toulouse, 31055 Toulouse, France \\ ${ }^{2}$ IES CNRS-UMR 5214, 34095 Montpellier, France \\ ${ }^{3}$ INA-Universidad de Zaragoza, 50018 Zaragoza, Spain
}

(Received 22 November 2013; accepted 10 January 2014; published online 23 January 2014)

\begin{abstract}
InAs/AlSb multilayers similar to those used in quantum cascade lasers have been grown by molecular beam epitaxy on (001) InAs substrates. Elastic strain is investigated by high resolution transmission electron microscopy. Thin interfacial regions with lattice distortions significantly different from the strain of the AlSb layers themselves are revealed from the geometrical phase analysis. Strain profiles are qualitatively compared to the chemical contrast of high angle annular dark field images obtained by scanning transmission electron microscopy. The strain and chemical profiles are correlated with the growth sequences used to form the interfaces. Tensile strained AlAs-like interfaces tend to form predominantly due to the high thermal stability of AlAs. Strongly asymmetric interfaces, AlAs-rich and (Al, In)Sb, respectively, can also be achieved by using appropriate growth sequences. (C) 2014 AIP Publishing LLC. [http://dx.doi.org/10.1063/1.4863035]
\end{abstract}

The InAs/AlSb material system is very attractive for the development of quantum cascade lasers (QCLs), in particular due to the large conduction band discontinuity of $2.1 \mathrm{eV}$, beneficial for fabrication of short wavelength QCLs, and a moderate lattice mismatch between these compounds. InAs/AlSb QCLs emitting in the mid-infrared range starting at wavelengths as short as $2.6 \mu \mathrm{m}$ have been demonstrated. ${ }^{1-5}$ The QCLs consist of a complex stack of very thin layers often including AlSb barriers thinner than a few monolayers to insure required coupling between adjacent InAs quantum wells. The thickness of each layer should be controlled with a sub-monolayer precision to provide the proper functioning of the device. The operation of InAs/AlSb QCLs is thus influenced by properties of interfaces between the constituting layers. InAs and AlSb have no atoms in common, so that interfacial zones necessarily include either Al-As bonds or In$\mathrm{Sb}$ bonds or a mix of them. It turns out that AlAs and InSb, as bulk materials, have a very large misfit with InAs, $-6.6 \%$ and $+6.9 \%$, respectively. While the lattice mismatch of AlSb with InAs is only $1.3 \%$, the interfaces themselves can be responsible for much larger local distortions, which can affect the device properties through a loss of structural quality and a modification of the band structure. ${ }^{6,7}$ In the $\mathrm{InAs} / \mathrm{GaSb}$ system, which presents a similar problematic, a tensile GaAslike interface has been very recently evidenced by transmission electron microscopy (TEM) ${ }^{8}$ The role of In-Sb interfacial bonds in morphological instabilities was also shown by synchrotron X-Ray diffraction. ${ }^{9}$ and cross-sectional scanning tunneling microscopy (XSTM). ${ }^{10}$ Intermixing at InAs/GaSb interfaces was also studied using Z-contrast microscopy ${ }^{11,12}$ and atomic probe tomography. ${ }^{13}$ In the InAs/AlSb system, some structural studies reported to-date concern mainly the interfacial roughness using $\mathrm{TEM}^{7,14}$ or interfacial intermixing using $\mathrm{XSTM}^{14}$ In a recent study, X-ray diffraction was used to study the effect of interfaces on the averaged strain in $\mathrm{AlSb} / \mathrm{InAs}$ superlattices on $\mathrm{GaSb}$ substrates; various growth sequences were applied in order to achieve full strain compensation. ${ }^{15}$ Nevertheless, very little is known about the actual strain state at interfaces, because of strong localization of these strains at the scale of 1 or 2 atomic layers. In this article, we focus on the strain generated by the interfaces themselves aiming at establishing the impact of the atomic bond configuration.

Multilayer structures alternating 20-nm-thick InAs and 4nm-thick AlSb layers have been grown on (001) InAs substrates by molecular beam epitaxy (MBE) at $700 \mathrm{~K}$ and a growth rate of $1 \mathrm{~A} / \mathrm{s}$. Antimony and arsenic valved cracker cells were used for growth providing a V/III flux ratio close to 2 both for AlSb and for InAs. We chose to grow samples with layers thicker than in QCLs to avoid superposition between displacement fields from adjacent interfaces (the value of $4 \mathrm{~nm}$ for AlSb appears as a suitable compromise to meet this objective without risk of plastic relaxation). In the first sample, named $\mathrm{A}$, the growth sequence at the $\mathrm{AlSb}$ on InAs and InAs on AlSb interfaces consisted in a simple growth interruption of $3 \mathrm{~s}$ under no element $\mathrm{V}$ flux. In the second sample, named B, we intended to favor formation of different types of interfaces by using the following growth sequence: [InAs + AlAs (2s) + stop (3s) + AlSb + InSb (2s) + stop $(3 \mathrm{~s})+\mathrm{InAs}]$. The $2 \mathrm{~s}$ growth duration corresponds to a deposit of $2 / 3$ of a monolayer. In this case we intended to favor formation of an AlAs-like interface on InAs and an InSb-like interface on top of the AlSb layer. The samples have been examined by high resolution electron microscopy (HREM) in a TECNAI F-20 microscope operating at $200 \mathrm{kV}$, equipped with a spherical aberration corrector allowing to avoid the delocalization effect at interfaces and to achieve a $0.12 \mathrm{~nm}$ spatial resolution. Samples have then been examined by Scanning Transmission Electron Microscopy (STEM) in high angle annular dark-field (HAADF) mode, using a TITAN Low-Base $300 \mathrm{kV}$, fitted with a Wien filter monochromator and a Cs probe corrector achieving a STEM spatial resolution of $0.08 \mathrm{~nm} \cdot{ }^{1-10}$ and 110 cross-sectional lamellas have been prepared by mechanical polishing and ion milling. 
Fig. 1(a) shows a HREM image of the sample A in the ${ }^{1-10}$ zone axis. Variations of the lattice fringes have been measured by applying the geometrical phase analysis (GPA). ${ }^{16}$ In this method the strains $\left(\varepsilon_{\mathrm{xx}}\right.$ and $\varepsilon_{\mathrm{yy}}$ in the following) are determined relatively to a reference zone, here chosen in the middle of an InAs layer, and therefore result from the addition of two contributions: the lattice mismatch $\mathrm{f}$ between InAs and the studied area, and the epitaxial strain undergone by the material ( $\varepsilon_{\text {phys }}$ in the following). The same analysis has been realized on different regions of one TEM lamella, then on different lamellas and along the two $\langle 110\rangle$ directions. Very similar results have been obtained.

The in-plane strain mapping $\varepsilon_{\mathrm{xx}}$ (Fig. 1(b)) revealed no significant variations, indicating full elastic accommodation
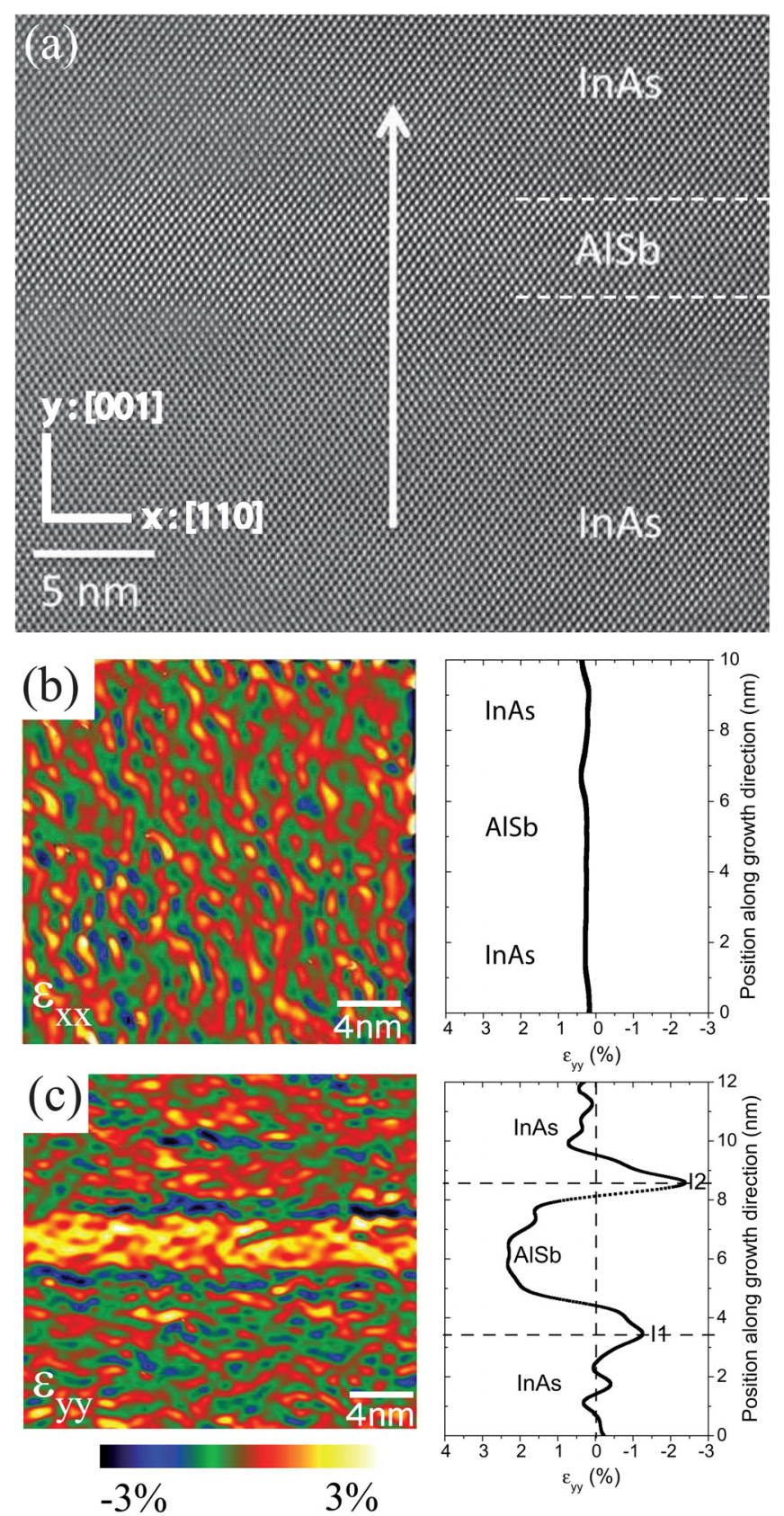

FIG. 1. (a) HREM image of InAs-AlSb superlattice along the [1-10] zone axis; (b) $\varepsilon_{\mathrm{xx}}$; and (c) $\varepsilon_{\mathrm{yy}}$ maps and profiles determined from the geometrical phase analysis of the HREM image $(0.8 \mathrm{~nm}$ spatial resolution). The profiles are averaged along the 110 direction. The arrows indicate the growth direction. of the lattice mismatch. The presence of the strained layer is clearly evidenced in the map of the out-of-plane strain $\varepsilon_{y y}$ (Fig. 1(c)). The $\varepsilon_{y y}$ profile along the growth axis is reported in Fig. 1(c) after being averaged over $33 \mathrm{~nm}$ along the in-plane direction 110. It reveals a plateau around $2.3 \%$. Considering images from different areas or lamellas, the values obtained for this plateau are between $2.3 \%$ and $3 \%$. This result has to be compared with the theoretical value calculated in the hypothesis of an elastic strain in a 001 oriented cubic system, following the well-known relationship:

$$
\varepsilon_{y y}=f+\varepsilon_{\text {phys }}^{\perp}=f\left[1+2 \frac{C_{12}}{C_{11}}\right],
$$

where $\mathrm{C}_{11}$ and $\mathrm{C}_{12}$ are the elastic coefficients (for $\mathrm{AlSb}$ $87.8 \mathrm{GPa}$ and $43.5 \mathrm{GPa}$, respectively). ${ }^{17} \mathrm{We}$ obtain $2.3 \%$, a value which has to be corrected by the thin foil effect. ${ }^{18}$ Considering this effect, in a 4-nm-thick strained layer embedded between two thick layers, $\varepsilon_{\text {yy }}$ measured is expected to be in the range $2.1 \%$ to $2.4 \%$ due to a reduction of the strain by $10 \%$ to $20 \% .{ }^{19}$ Although, sometimes, slightly larger than expected, the measured strain is therefore fully consistent with the presence of an elastically strained AlSb layer. In addition to the plateau, the out-of-plane strain profile exhibits two negative peaks (I1 and I2 in Fig. 1(c)), respectively, of $-1.5 \%$ and $-2.5 \%$ at the first and the second interfaces, suggesting the formation of two tensile strained interfaces. Considering images from different areas of lamellas, the values obtained for the two negative peaks exhibits quite large fluctuations from $-1 \%$ to $-6 \%$. As these peaks are significantly larger than expected from the classical transfer of stress to substrate due to the thin foil effect, ${ }^{19}$ it is tentatively attributed to the preferential formation of $\mathrm{Al}$-As bonds at both the AlSb on InAs and InAs on AlSb interfaces. Theoretical $\mathrm{f}$ for AlAs on InAs being -6.6\%, applying (1) results in $\varepsilon_{y y}$ of $-13 \%$. In addition to a possible thin foil effect, this large difference with the measured values can be explained by an effect of the GPA treatment specific to ultra-thin layers. Indeed, a mask is used in the reciprocal space of the HREM image to select the lattice fringes to analyze. The spatial resolution of the strain profile is inversely related to the mask size; here this resolution is $0.8 \mathrm{~nm}$, i.e., greater than the supposed thickness of the interface, which is closer to two atomic planes. Thus, the GPA method gives a strain value averaged over a larger zone than the actual interface. Moreover, the formation of a ternary or quaternary compound less strained than AlAs can also explain this difference. HRSTEM images have been realized on the same sample in HAADF mode. The GPA treatment could not be used on these images because the InAs dumbbells are resolved whereas the AlSb ones, due to the large difference between the atomic numbers ( $\mathrm{Z}$ ) of $\mathrm{Al}$ and $\mathrm{Sb}$, are not resolved. Therefore, an artificial phase jump appears at the interfaces, unlike in HREM images made so as to be uniform over the whole image. HAADF images were thus exploited to get qualitative chemical information: In HAADF mode, the scattered intensity increases with the $\mathrm{Z}$ of the material (reported in Table I for the bulk binaries). The intensity profile from the HAADF image (Fig. 2) shows a symmetric shape, with a clear signature of the AlSb layer embedded 
TABLE I. Structural and thermodynamic properties of the binary bulk compounds. ${ }^{17}$

\begin{tabular}{lcccc}
\hline \hline Crystal & $\begin{array}{c}\text { Melting } \\
\text { point (K) }\end{array}$ & $\begin{array}{c}\text { Cohesive } \\
\text { energy (eV) }\end{array}$ & $\begin{array}{c}\text { Misfit f with } \\
\text { InAs (\%) }\end{array}$ & $\begin{array}{c}\text { Average atomic } \\
\text { number Z }\end{array}$ \\
\hline AlAs & 2013 & 6.8 & -6.6 & 23 \\
AlSb & 1338 & 6.6 & 1.3 & 32 \\
InAs & 1215 & 5.4 & 0 & 41 \\
InSb & 800 & 4.7 & 6.9 & 50 \\
\hline \hline
\end{tabular}

between two interfaces looking significantly darker, which seems to indicate the formation of a lighter material.

This result indicates, in a good agreement with the strain analysis, that the spontaneously formed interfaces have preferentially Al-As bonds. A possible explanation is that the AlAs bonds are the most stable among the four possible bonds configurations in this system (in bulk materials), and in particular they are much more stable than In-Sb bonds, the other possible candidate at interfaces (cf. Table I).

Similar GPA treatment has been realized on several HREM images of the B sample in both [1-10] and [110] zone axis. A representative example is displayed in Fig. 3. Although less marked than previously, a small plateau (between $2 \%$ and $3 \%$ ) corresponding to AlSb is still visible (marked as 2 in Fig. 3(b)).

A very interesting point is that the out-of-plane strain map now exhibits an asymmetric profile with a negative peak at the first interface and a positive peak at the second interface, (respectively, 1 and 3 in Fig. 3(b)). The negative peak at $-8 \%$ seems to indicate the preferential formation of highly tensile strained bonds (AlAs-like type) while the positive peak at $7 \%$ indicates the formation of highly compressive strained bonds (InSb-like type). The formation of asymmetric interfaces is also confirmed by HRSTEMHAADF analysis (Fig. 4). Indeed, the first interface appears to be significantly darker than the AlSb layer indicating the formation of an AlAs-rich layer. The scattered intensity at the second interface increases continuously between those of the AlSb and InAs layers. This corresponds to an average composition heavier than AlSb but lighter than InAs. Two ternary alloys, $\mathrm{Al}_{\mathrm{x}} \mathrm{In}_{1-\mathrm{x}} \mathrm{Sb}(\mathrm{x}>0.5)$ and $\mathrm{Al}_{\mathrm{y}} \mathrm{In}_{1-\mathrm{y}} \mathrm{As}(\mathrm{y}<0.5)$, are possible candidates (from Table I). Nevertheless, $\mathrm{Al}_{\mathrm{x}} \mathrm{In}_{1-\mathrm{x}} \mathrm{Sb}$ corresponds effectively to the average interfacial composition expected from the growth sequence, while $\mathrm{Al}_{\mathrm{y}} \mathrm{In}_{1-\mathrm{y}} \mathrm{As}$ seems much less likely. In the same time,
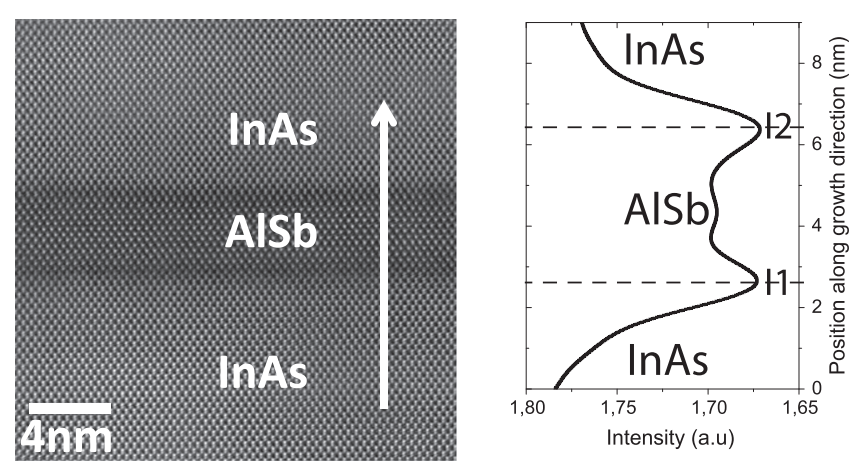

FIG. 2. HAADF image on one AlSb layer of a sample and intensity profile along the growth direction.
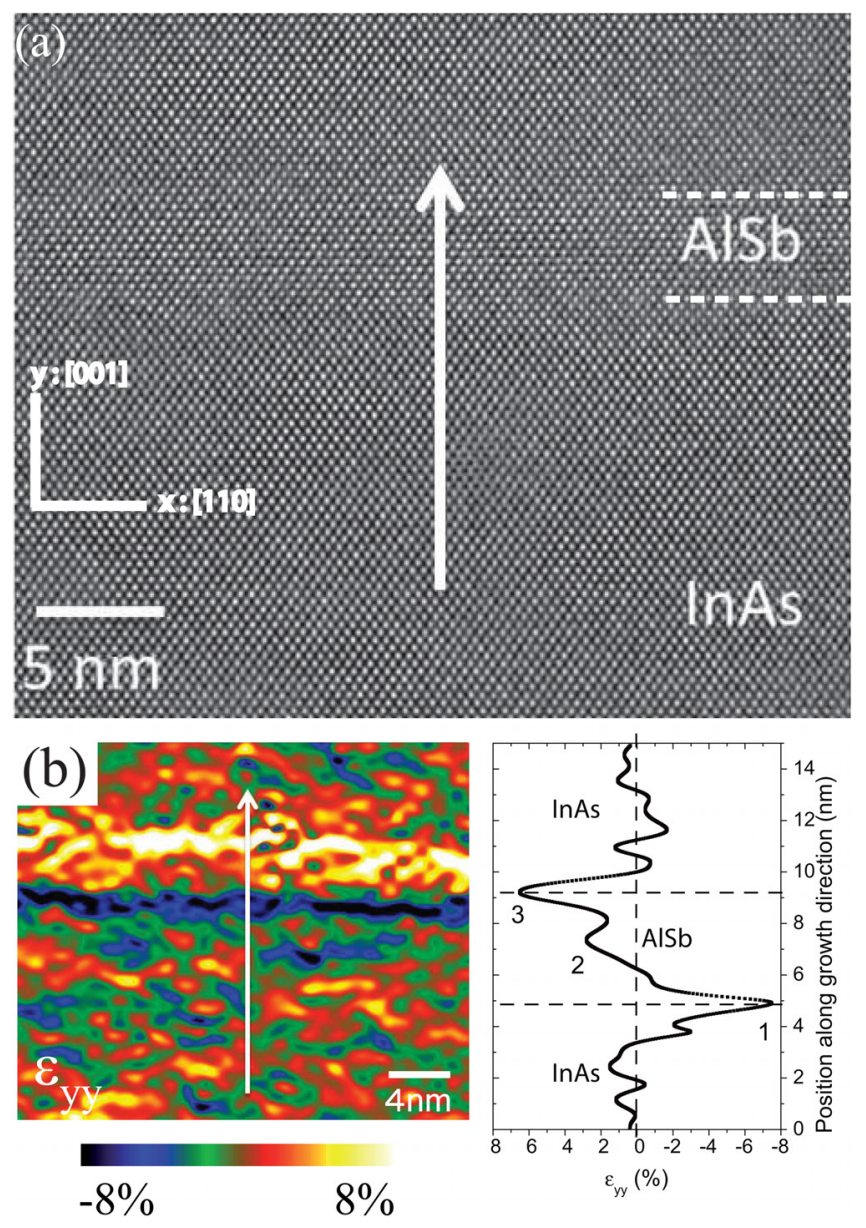

FIG. 3. (a) High resolution TEM image of InAs-AlSb superlattice realized on the sample B, along the ${ }^{1-10}$ zone axis; (b) $\varepsilon_{\mathrm{yy}}$ map and profile determined from the geometrical phase analysis $(0.8 \mathrm{~nm}$ spatial resolution). The arrow indicates the growth direction.

$\mathrm{Al}_{\mathrm{y}} \mathrm{In}_{1-\mathrm{y}} \mathrm{As}$ presents a negative misfit with InAs (in the whole range of $y$ ) and thus is not compatible with the strain analysis. On the contrary, $\mathrm{Al}_{\mathrm{x}} \mathrm{In}_{1-\mathrm{x}} \mathrm{Sb}(\mathrm{x}>0.5)$ is fully consistent with the strain profile, its misfit with InAs varying between $1.3 \%$ (AlSb) and $4.1 \%\left(\mathrm{Al}_{0.5} \mathrm{In}_{0.5} \mathrm{Sb}\right)$. The HAADF profiles are thus in very good agreement with the GPA analysis. Note that this apparent average composition can be due to either an actual mixing along the growth direction, or to lateral fluctuations.

The obtained results are very helpful for optimization of InAs/AlSb QCLs. Our data demonstrates that compressive
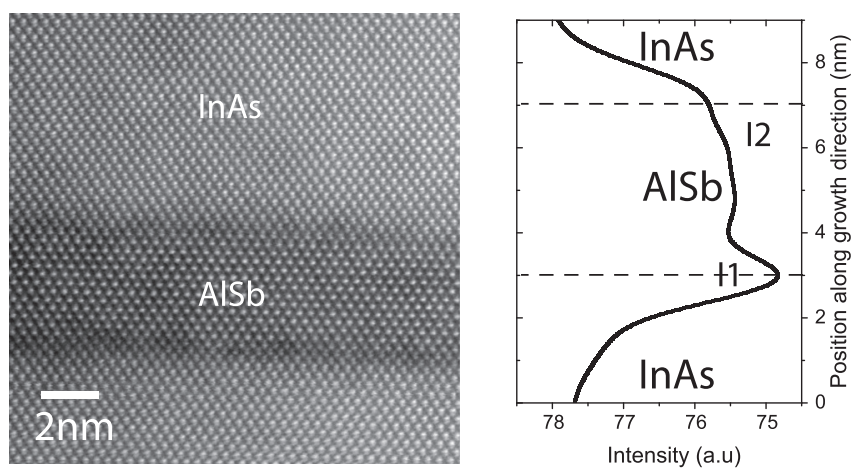

FIG. 4. HRSTEM-HAADF image and intensity profile associated of sample B. The arrow indicates the growth direction. 
strains induced by AlSb in QCLs grown on InAs can be compensated by spontaneously forming abrupt AlAs-rich interfaces. When an InAs/AlSb QCL is grown on a GaSb substrate its InAs layers induce tensile strains in the structure. This work, suggests that formation of InSb-rich interfaces, needed to avoid plastic relaxation in the QCLs grown on $\mathrm{GaSb}$, requires special growth sequences including forced deposition of InSb. The obtained data can be employed to estimate the interface effect on the total strain in the QCL and to correct it by modification of the MBE growth conditions. It also gives indications how to take into account the interface effect on the QCL electronic structure in the device modeling.

To conclude, the impact of interfaces on strain profiles has been directly evidenced at a local scale by the GPA method applied to HREM images. It is shown that ultrathin interfacial layers are effectively formed due to the fact than InAs and AlSb have no atoms in common. They present a lattice distortion different than the one due to the average mismatch between InAs and AlSb. Chemical compositions, analyzed from STEM-HAADF at the interfacial scale, are in very good agreement with the GPA analysis. Combining STEM-HAADF and GPA, it has been possible to determine more precisely the chemical composition of the interfaces than using only one of these methods. We observed that the strain status of these layers depends on the growth sequence used to form the interfaces. Tensile strained AlAs-like interfaces tend to form predominantly both on InAs and on AlSb due to the higher thermal stability of AlAs compared with other binary compounds that can exist at the layer boundaries in this material system. However, the formation of strongly asymmetric interfaces, tensile AlAs-rich and compressive (Al, In)Sb, respectively, can also be achieved by using appropriate growth sequences. The actual interfacial composition will be discussed in more details in a forthcoming paper, as well as the effects of various growth sequences.
The authors are grateful to C. Crestou for TEM samples thinning. They also acknowledge the CNRS and ESTEEM 2 (Enabling Science and Technology through European Electron Microscopy) for supporting TEM-STEM experiments. This work is supported by the French national project NAIADE (ANR-11-BS10-017).

${ }^{1}$ J. Devenson, O. Cathabard, R. Teissier, and A. N. Baranov, Appl. Phys. Lett. 91(14), 141106 (2007).

${ }^{2}$ J. Devenson, O. Cathabard, R. Teissier, and A. N. Baranov, Appl. Phys. Lett. 91(25), 251102 (2007).

${ }^{3}$ O. Cathabard, R. Teissier, J. Devenson, J. C. Moreno, and A. N. Baranov, Appl. Phys. Lett. 96, 141110 (2010).

${ }^{4}$ P. Laffaille, J. C. Moreno, R. Teissier, M. Bahriz, and A. N. Baranov, AIP Adv. 2, 022119 (2012).

${ }^{5}$ M. Bahriz, G. Lollia, P. Laffaille, A. N. Baranov, and R. Teissier, Electron Lett. 49(19), 1238 (2013).

${ }^{6}$ G. Tuttle, H. Kroemer, and J. H. English, J. Appl. Phys. 67(6), 3032 (1990).

${ }^{7}$ J. Spitzer, A. Hopner, M. Kuball, M. Cardona, B. Jenichen, H. Neuroth, B. Brar, and H. Kroemer, J. Appl. Phys. 77(2), 811 (1995).

${ }^{8}$ K. Mahalingam, H. J. Haugan, G. J. Brown, and K. G. Eyink, Ultramicroscopy 127, 70 (2013).

${ }^{9}$ J. H. Li, D. W. Stokes, O. Caha, S. L. Ammu, J. Bai, K. E. Bassler, and S. C. Moss, Phys. Rev. Lett. 95(9), 096104 (2005).

${ }^{10}$ B. Z. Nosho, B. R. Bennett, L. J. Whitman, and M. Goldenberg, Appl. Phys. Lett. 81(23), 4452 (2002).

${ }^{11}$ E. Luna, F. Ishikawa, B. Satpati, J. B. Rodriguez, E. Tournié, and A. Trampert, J. Cryst. Growth 311(7), 1739 (2009).

${ }^{12}$ B. Satpati, J. B. Rodriguez, A. Trampert, E. Tournié, A. Joullié, and P. Christol, J. Cryst. Growth 301-302, 889 (2007).

${ }^{13}$ H. Kim, Y. Meng, J. L. Rouvière, D. Isheim, D. N. Seidman, and J. M. Zuo, J. Appl. Phys. 113, 103511 (2013).

${ }^{14}$ W. Barvosa-Carter, M. E. Twigg, M. J. Yang, and L. J. Whitman, Phys. Rev. B 63(24), 245311 (2001).

${ }^{15}$ A. Bauer, M. Dallner, A. Herrmann, T. Lehnhardt, M. Kamp, S. Höfling, L. Worschech, and A. Forchel, Nanotechnology 21(45), 455603 (2010).

${ }^{16}$ M. J. Hytch, E. Snoeck, and R. Kilaas, Ultramicroscopy 74(3), 131 (1998).

${ }^{17} \mathrm{~V}$. Swaminathan and A. T. Macrander, Materials Aspects of GaAs and InP based Structures (Prentice-Hall, Inc., 1991).

${ }^{18}$ M. M. J. Treacy and J. M. Gibson, J. Vac. Sci. Technol. B 4(6), 1458 (1986).

${ }^{19}$ C. Gatel, H. Tang, C. Crestou, A. Ponchet, N. Bertru, F. Doré, and H. Folliot, Acta Materialia 58(9), 3238 (2010). 